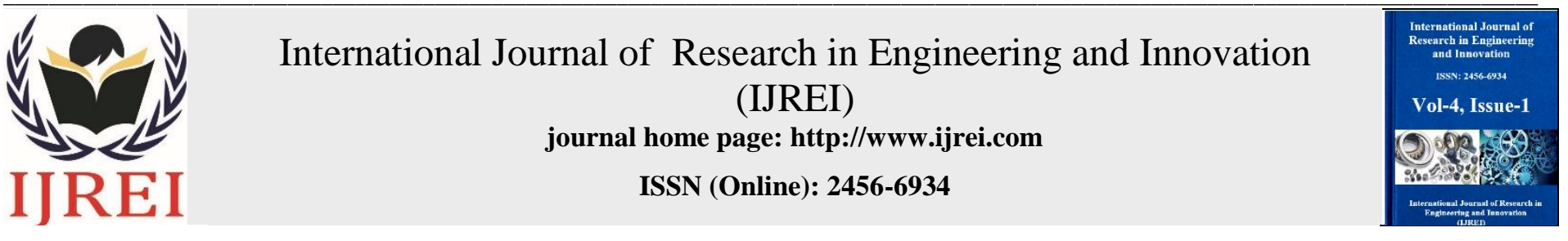

\title{
Numerical analysis and fluid structure interaction of flow around a flat plate at low reynolds number
}

\author{
Abhishek Yadav, Arshad Mehmood \\ ${ }^{1}$ Department of Mechanical Engineering, Roorkee College of Engineering, Roorkee, Uttrakhand, India \\ ${ }^{2}$ Department of Mechanical Engineering, College of Engineering/ University of Buraimi, Oman.
}

\begin{abstract}
Aerodynamic performance and fluid structure interaction (FSI) has been investigated via numerical evaluation of flow round a rectangular plate. This case is representing the complicated structure used in aerospace and surveillance in navy region. This analysis carried out via applying finite thing method over the fluid structure system of rectangular plate (structure) and fluid (air) with the aid of considering the coupled effect. The mathematical mannequin was once developed the use of a combination of FEM and sanders shell theory. The pace conceivable and Bernoulli's equation are adopted to categorical the fluid strain performing on the flat plate. In the first phase of the work fluid flow simulation at Reynold's number 500,1000, 2000, and 3000 will be performed with perspective of attack zero to one hundred fifty The end result from the CFD solver will be fed in the shape of carry and drag forces are fed into the ANSYS workbench solver and one way fluid shape interplay was performed The outcomes provide a great measure of confidence in the fidelity of the simulation (C)2020 ijrei.com. All rights reserved
\end{abstract}

Keywords: Drag Coefficient, Lift Coefficient, Reynolds Number, Angle of attack

\section{Introduction}

Fluid shape interplay of plates have been widely used in a number engineering discipline such as aircraft construction, present day construction engineering and nuclear strength plant etc. Many scientist have labored on vibration of such plates [1-2]. Classical plate idea (CPT) have been used with the aid of the many researchers. The herbal frequencies of the plate by using ignoring the shear deformation used to be investigated. The CPT was once proposed first order shear deformation plate concept to cast off the deficiency of the slightly thick plates [3-6]. The precise closed structure attribute equation of vibrating relatively thick rectangular plates was once investigated [7]. This strategy offers non-conservative results, so it is required to look at the fluid shape interplay (FSI) issues in a coupled manner considering the flexibility effect of the structure, so each the systems are coupled and solved as one device [8-11]. Formulation based totally on displacement variable are commonly chosen for the structure while the fluid is described through unique variables such as pressure, displacement, speed plausible etc. for such coupled problems. A range of investigator used hydrodynamic pressure as the unknown variable in FEM in the fluid area [12-13]. But in this case unsymmetrical matrices and unique cause laptop software are required [14-15]. The equations of fluid area in terms of a displacement workable was represented [16]. The coupled equations of movement turn out to be unsymmetrical but irrationality condition of fluid action is automatically satisfied. Many scientist have formulated governing equations of fluid in phrases of displacements. The major gain of the displacement primarily based components is that the fluid factors can be easily coupled with the structural factors the usage of FEM methods. But the degree of freedom for the fluid domain increases. Furthermore, the fluid displacement should satisfy the irrationality condition, otherwise zero frequency specious modes may also occur. The variable such as strain and speed used for representing the governing equations for fluid [9], but the requirement of computational time will become higher as variety of unknown parameters make bigger in the fluid domain. The answer of the coupled device might also be trained with the aid of fixing the two system one at a time with the interplay effects with the aid of iteration [20-24]. In contact with water, the first bending mode structure of a circular plate constant at 
circumference was once calculated [25]. Powell and Robert tested lamb's result by way of experimentally, they suggests that their frequencies had been slightly greater than the lamb [26]. The response of cantilever plate contact with air and water was carried out via the experimental techniques [27] and these consequences had been in contrast with theoretical calculations. The natural frequencies of vertical cantilever plates in part and definitely submerged in liquid was calculated. These values have been in contrast with outcomes acquired with the aid of finite component methods. The herbal frequency bought with the aid of finite element techniques were about $15 \%$ higher than those got in experiments [28]. The vibration response of cantilever vertical and horizontal plates in part or totally submerge in water used to be studied. It used to be analyzed that the plates vibrated in a semi-infinite fluid medium. They used an aggregate of finite aspect technique (FEM) and singularity distribution panel approach to find out the dynamic responses of plates in vacuum [29]. The fluid Structure Interaction of drift and aerodynamic overall performance of two dimensional pleated airfoil is carried out at Reynolds range 100, 200, 500, and one thousand will be performed with perspective of assault zero to 150 [30-33]. The coefficient of drag, pressure distribution and vortex shedding for different Reynolds variety with exclusive attitude of assault had been analyzed and in contrast with numerical result that shows desirable agreement [34-35]

\section{Numerical Methodology [40]}

\subsection{Structural Model}

Fig. $1 \mathrm{~b}$ indicates the four node element and nodal diploma of freedom. Each node has six diploma of freedom that suggests the in-plane and out-plane displacement factors and their spatial derivatives. Sander's thin shell principle [36] gives zero strain for small inflexible physique motion, this case is no longer same as other theories [37-39]. To advance the governing equations of the rectangular plates, the sander's equation for cylindrical shells are used assuming the radius to be infinite, i.e. two $\theta=y$ and $r d \theta=d y$.

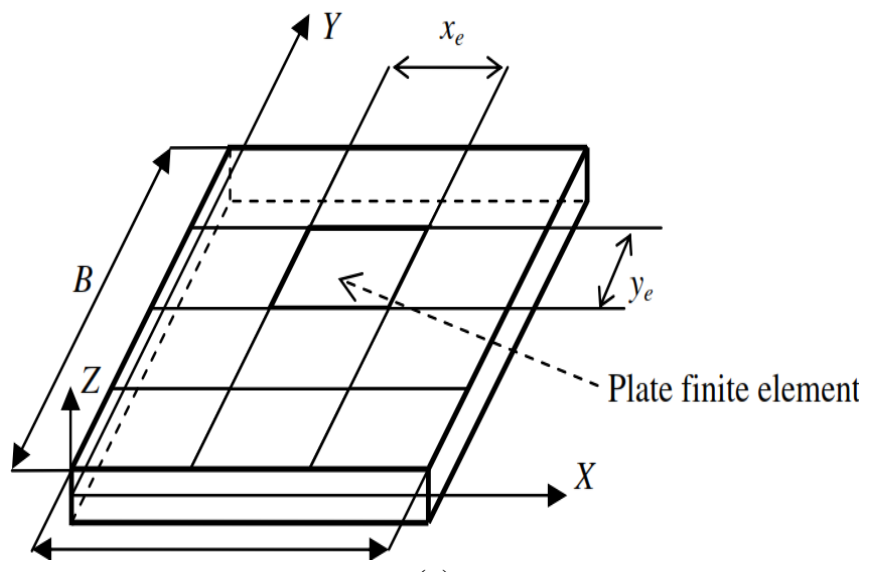

(a)

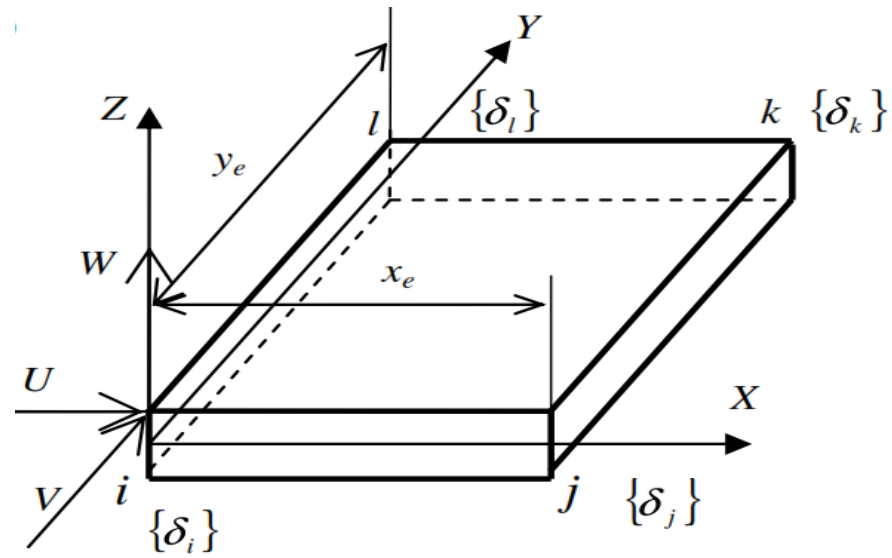

(b)

Figure 1: (a) Finite aspect discretization of rectangular plate and (b) Geometry and displacement field of a normal issue [40]

\subsection{Equilibrium equation and displacement function [40]}

$\mathrm{P}_{22} \frac{\partial^{2} V}{\partial y^{2}}+\mathrm{P}_{21} \frac{\partial^{2} U}{\partial x \partial y}+\mathrm{P}_{33}\left(\frac{\partial^{2} U}{\partial x \partial y}+\frac{\partial^{2} V}{\partial x^{2}}\right)=0$

$\mathrm{P}_{11} \frac{\partial^{2} U}{\partial x^{2}}+\mathrm{P}_{12} \frac{\partial^{2} V}{\partial x \partial y}+\mathrm{P}_{33}\left(\frac{\partial^{2} V}{\partial x \partial y}+\frac{\partial^{2} U}{\partial x^{2}}\right)=0$

$\mathrm{P}_{44} \frac{\partial^{4} W}{\partial x^{4}}+\left(\mathrm{P}_{45}+\mathrm{P}_{54}+2 \mathrm{P}_{66}\right) \frac{\partial^{4} W}{\partial x^{2} \partial y^{2}}+\mathrm{P}_{55} \frac{\partial^{4} W}{\partial y^{4}}=0$

The displacement field may be defined as follows:

$U(x, y, t)=\mathrm{C}_{1}+\mathrm{C}_{2} \frac{\mathrm{x}}{\mathrm{A}}+\mathrm{C}_{3} \frac{\mathrm{y}}{\mathrm{B}}+\mathrm{C}_{4} \frac{\mathrm{xy}}{\mathrm{AB}}$
$V(x, y, t)=\mathrm{C}_{5}+\mathrm{C}_{6} \frac{\mathrm{x}}{\mathrm{A}}+\mathrm{C}_{7} \frac{\mathrm{y}}{\mathrm{B}}+\mathrm{C}_{8} \frac{\mathrm{xy}}{\mathrm{AB}}$
$W(x, y, t)=\sum_{9}^{24} C_{j} e^{i \pi\left(\frac{x}{A}+\frac{y}{B}\right)} e^{i \omega t}$

The membrane effects emerge as extraordinarily important. Eq. (6) can be developed in Taylor's series as [41]

$$
\begin{aligned}
& W(x, y, t)=\mathrm{C}_{9}+\mathrm{C}_{10} \frac{\mathrm{x}}{\mathrm{A}}+\mathrm{C}_{11} \frac{y}{B}+\mathrm{C}_{12} \frac{\mathrm{x}^{2}}{2 A^{2}}+\mathrm{C}_{13} \frac{\mathrm{xy}}{A B}+\mathrm{C}_{14} \frac{\mathrm{y}^{2}}{2 B^{2}}+\mathrm{C}_{15} \frac{\mathrm{x}^{3}}{6 A^{3}}+ \\
& \mathrm{C}_{16} \frac{\mathrm{x}^{2} y}{2 A^{2} B}+\mathrm{C}_{17} \frac{\mathrm{xy}^{2}}{2 A B^{2}}+\mathrm{C}_{18} \frac{y^{3}}{6 B^{3}}+\mathrm{C}_{19} \frac{\mathrm{x}^{3} y}{6 A^{3} B}+\mathrm{C}_{20} \frac{\mathrm{x}^{2} \mathrm{y}^{2}}{4 A^{2} B^{2}}+\mathrm{C}_{21} \frac{\mathrm{x}^{3} \mathrm{y}^{2}}{6 A B^{3}}+ \\
& \mathrm{C}_{22} \frac{\mathrm{x}^{3} \mathrm{y}^{2}}{12 A^{3} B^{2}}+\mathrm{C}_{23} \frac{\mathrm{x}^{2} \mathrm{y}^{3}}{12 A^{2} B^{3}}+\mathrm{C}_{24} \frac{\mathrm{x}^{3} \mathrm{y}^{3}}{36 A^{2} B^{3}}
\end{aligned}
$$

The displacement field may additionally be rewritten in the form of matrix members of the family as follows

$$
\begin{aligned}
& \left\{\begin{array}{c}
U \\
V \\
W
\end{array}\right\}=[\mathrm{R}]\{\mathrm{C}\} \\
& \{\mathrm{C}\}=\left\{\mathrm{C}_{1}, \mathrm{C}_{2} \ldots \ldots \ldots . \mathrm{C}_{24}\right\}^{\mathrm{T}}
\end{aligned}
$$

The factors of this final vector can be determined the use of 24 levels of freedom introduced for a plate thing as shown in Fig. 1b. The displacement vector of every component is given as 
$\{\delta\}=\left\{\left\{\delta_{\mathrm{i}}\right\}^{\mathrm{T}},\left\{\delta_{\mathrm{j}}\right\}^{\mathrm{T}},\left\{\delta_{\mathrm{k}}\right\}^{\mathrm{T}},\left\{\delta_{\mathrm{l}}\right\}^{\mathrm{T}}\right\}$

Each node, i.e. "'node i', possesses a nodal displacement vector composed of the following terms:

$\left\{\delta_{\mathrm{i}}\right\}=\left\{\mathrm{U}_{\mathrm{i}}, \mathrm{V}_{\mathrm{i}}, \mathrm{W}_{\mathrm{i}}, \partial \mathrm{W}_{\mathrm{i}} / \partial \mathrm{x}, \partial \mathrm{W}_{\mathrm{i}} / \partial \mathrm{y}, \partial^{2} \mathrm{~W}_{\mathrm{i}} / \partial \mathrm{x} \partial \mathrm{y}\right.$

The elementary displacement vector can be defined as

$\{\delta\}=[\mathrm{A}]\{\mathrm{C}\}$

where $[\mathrm{A}]$ is a $(24,24)$ matrix.

The displacement field may be described by the following relation:

$\left\{\begin{array}{l}U \\ V \\ W\end{array}\right\}=[\mathrm{R}][\mathrm{A}]^{-1}\{\delta\}=[\mathrm{N}]\{\delta\}$

Where matrix $[\mathrm{N}]$ of order $(3 \times 24)$ is the displacement shape function of the finite element.

\subsection{Kinematics relations}

$\left\{\begin{array}{c}\varepsilon_{x} \\ \varepsilon_{y} \\ 2 \varepsilon_{x y} \\ K_{x} \\ K_{y} \\ K_{x y}\end{array}\right\}=\left\{\begin{array}{c}\frac{\partial U}{\partial x} \\ \frac{\partial V}{\partial y} \\ \frac{\partial V}{\partial x}+\frac{\partial U}{\partial y} \\ -\frac{\partial^{2} W}{\partial x^{2}} \\ -\frac{\partial^{2} W}{\partial y^{2}} \\ -2 \frac{\partial^{2} W}{\partial x \partial y}\end{array}\right\}$

Substituting the displacement elements defined in Eq. (13) into the strain-displacement relationship (14), one obtains an expression for the pressure vector as a feature of nodal displacements.

$\varepsilon=[\mathrm{Q}][\mathrm{A}]^{-1}\{\delta\}=[\mathrm{B}]\{\delta\}$

Where matrix [Q], of order (6x24)

\subsection{Constitutive equations}

The stress-strain relationship of an anisotropic rectangular plate is defined as follows.

$\{\sigma\}=[\mathrm{P}]\{\varepsilon\}$

Where $[\mathrm{P}]$ is the elasticity matrix $(6 \times 6)$. The elements of $[\mathrm{P}]$ symbolize the shell anisotropy, Substituting Eq. (15) into Eq. (16) results in the following expression for the stress vector as a function of nodal displacements [42-49].

$\{\sigma\}=[\mathrm{P}][\mathrm{B}]\{\delta\}$

The mass and stiffness matrices for one finite element can be expressed as

$\left[K_{S}\right]^{e}=\iint_{A}[B]^{T}[P][B] d A$

$\left[m_{s}\right]^{e}=\rho_{s} h \iint_{A}[N]^{T}[N] d A$

Where $\mathrm{dA}$ is the issue surface area, $\mathrm{h}$ is the plate thickness and $\rho \_s$ is the material density and $[\mathrm{P}],[\mathrm{N}]$ and $[\mathrm{B}]$ are defined in Eqs. (16, 13 and 15), substituting them into Eqs. (18.a and 18.b) we attain

$\left[K_{s}\right]^{e}=\left[[A]^{-1}\right]^{T}[\mathrm{~A}]\left(\int_{0}^{y_{c}} \int_{0}^{x_{c}}[Q]^{T}[P][Q] d x d y\right)[\mathrm{A}]^{-1} \quad(19 \mathrm{a})$

$\left[m_{s}\right]^{e}=\rho_{s} h\left[[A]^{-1}\right]^{T}[\mathrm{~A}]\left(\int_{0}^{y_{c}} \int_{0}^{x_{c}}[Q]^{T}[P][Q] d x d y\right)[\mathrm{A}]^{-1}(19 \mathrm{~b})$

Where $\mathrm{xc}$ and $\mathrm{yc}$ are dimensions of an issue in accordance to the $\mathrm{X}$ and $\mathrm{Y}$ coordinates, respectively. These integrals are calculated the use of Maple mathematical software

\section{Fluid Modeling}

Linear potential flow is applied to shows the fluid effect that leads to the fluid dynamic forces. The Laplace equation may be expressed as in the Cartesian coordinate

$\nabla^{2} \varphi=\frac{\partial^{2} \varphi}{\partial x^{2}}+\frac{\partial^{2} \varphi}{\partial y^{2}}+\frac{\partial^{2} \varphi}{\partial z^{2}}=0$

Using Bernoulli's equation the fluid pressure at the solid-fluid interface may be expressed as

$\left.P\right|_{\mathrm{z}=0}=-\left.\rho_{f} \frac{\partial \varphi}{\partial t}\right|_{\mathrm{z}=0}$

The following separate variable relation is assumed for the potential velocity function

$\varphi(\mathrm{x}, \mathrm{y}, \mathrm{z}, \mathrm{t})=\mathrm{F}(\mathrm{z}) \mathrm{S}(\mathrm{x}, \mathrm{y}, \mathrm{t})$

Where $F(z)$ and $S(x, y, z)$ are two separate functions to be determined.

The permanent contact between the plate surface and the peripheral fluid layer may be written as

$\left.\frac{\partial \varphi}{\partial z}\right|_{z=0}=\frac{\partial W}{\partial t}$ 
The following expression may be defined by introducing Eq. (16) into Eq. (17)

$\mathrm{S}(\mathrm{x}, \mathrm{y}, \mathrm{t})=\frac{1}{d F(0) / d z} \frac{\partial W}{\partial t}$

For $\mathrm{X}$ and $\mathrm{Y}$ in the finite element domain (see Figs. 1b and 2), substituting Eq. (24) into (22), and results in the following expression for the potential function:

$\varphi(\mathrm{x}, \mathrm{y}, \mathrm{z}, \mathrm{t})=\frac{F(\mathrm{z})}{d F(0) / d z} \frac{\partial W}{\partial t}$

Substituting Eq. (25) into relation (20) leads to the following differential equation of second order.

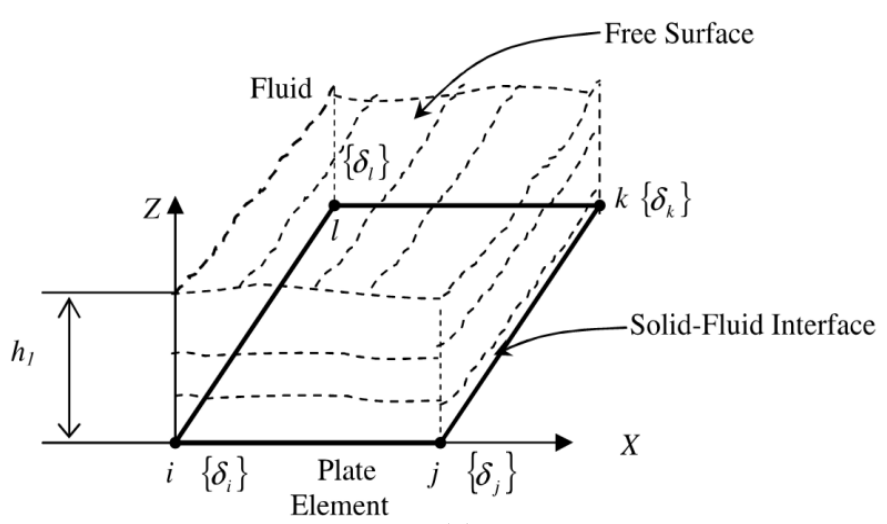

(a)

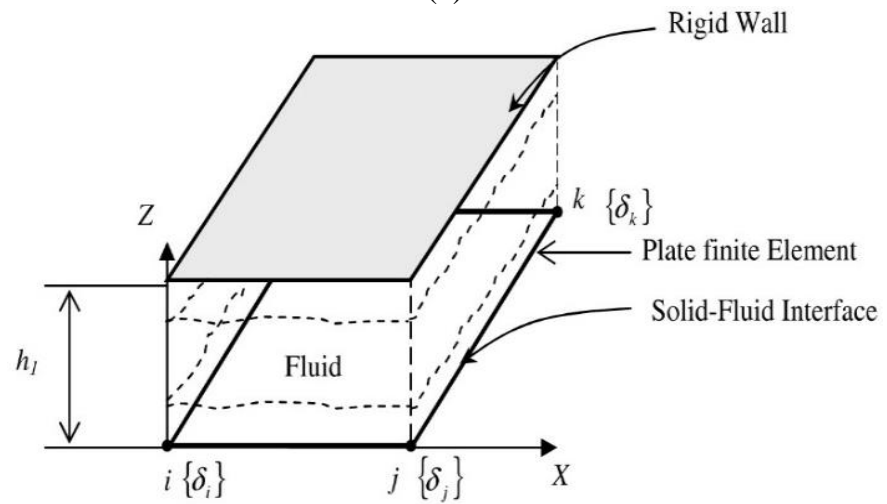

(b)

Figure 2. (a) Coupled fluid-structure element possessing a free surface of fluid at $Z=h_{1}$ and (b) plate element in contact with fluid bounded by a rigid wall at $Z=h_{1}[40]$

$\frac{d^{2} F(z)}{d z^{2}}-\mu^{2} F(z)=0$

Where $\mu=\pi \sqrt{\frac{1}{A^{2}}+\frac{1}{B^{2}}}$

The general solution of eq. (26) is given as

$\mathrm{F}(\mathrm{z})=\mathrm{A}_{1} \mathrm{e}^{\mu \mathrm{z}}+\mathrm{A}_{2} \mathrm{e}^{-\mu \mathrm{z}}$

Substituting eq. (27) into (25), one gets the following expression for the potential function.

$\varphi(\mathrm{x}, \mathrm{y}, \mathrm{z}, \mathrm{t})=\frac{\left(A_{1} e^{\mu \mathrm{z}}+A_{2} e^{-\mu \mathrm{z}}\right)}{d F(0) / d z} \frac{\partial W}{\partial t}$

Where $\mathrm{A}_{1}$ and $\mathrm{A}_{2}$ are two unknown constants. The potential function $\varphi$ must be verified for given boundary conditions at the fluid-structure interface and the fluid extremity surfaces $\left(Z=h_{1}\right.$ or $\mathrm{Z}=\mathrm{h}_{2}$ ) as well.

\subsection{Plate fluid model with free surface}

The following condition may be applied at the fluid free surface to the velocity potential, see Fig. $2 \mathrm{a}$

$\left.\frac{\partial \varphi(x, y, z, t)}{\partial z}\right|_{\mathrm{z}=\mathrm{h} 1}=-\frac{1}{g} \frac{\partial^{2} \varphi}{\partial t^{2}}$

The introduction of Eq. (28) simultaneously into relation (29) and (23), results in the following expression for the potential function

$\varphi(\mathrm{x}, \mathrm{y}, \mathrm{z}, \mathrm{t})=\frac{1}{\mu}\left[\frac{e^{\mu \mathrm{z}}+C e^{-\mu\left(\mathrm{z}-2 h_{1}\right)}}{1-C e^{2 \mu h_{1}}}\right] \frac{\partial W}{\partial t}$

Where $C=\left(g \mu-\omega^{2}\right) /\left(g \mu+\omega^{2}\right)$

The corresponding dynamic pressure at the fluid-structure interface become

$\mathrm{P}=\frac{\rho_{f}}{\mu}\left[\frac{1+C e^{2 \mu h_{1}}}{1-C e^{2 \mu h_{1}}}\right] \frac{\partial^{2} W}{\partial t^{2}}=\mathrm{Z}_{\mathrm{f} 1} \frac{\partial^{2} W}{\partial t^{2}}$

\subsection{Plate-fluid model bounded by a rigid wall}

The boundary condition at the upper surface of the fluid represented in Fig. 2b was studied by Lamb [25] and referred to as the null-frequency condition. This rigid wall boundary condition is expressed as

$$
\left.\frac{\partial \varphi}{\partial z}\right|_{\mathrm{z}=\mathrm{h} 1}=0
$$

Similarly, by introducing Eq. (28) into relations (33) and (23), we obtain the following expression for the velocity potential as follows

$\varphi(\mathrm{x}, \mathrm{y}, \mathrm{z}, \mathrm{t})=\frac{1}{\mu}\left[\frac{e^{-\mu \mathrm{z}}+e^{\mu\left(\mathrm{z}-2 h_{1}\right)}}{e^{-2 \mu h_{1}}-1}\right] \frac{\partial W}{\partial t}$

The dynamic pressure for this case is determined as

$\mathrm{P}=-\frac{\rho_{f}}{\mu}\left[\frac{e^{-2 \mu h_{1}}+1}{e^{-2 \mu h_{1}}-1}\right] \frac{\partial^{2} W}{\partial t^{2}}=\mathrm{Z}_{f 2} \frac{\partial^{2} W}{\partial t^{2}}$

The total dynamic pressure is therefore the sum of lower and upper pressures (fig.3) and can be calculated using Eqs. (32) and 
(35), respectively. The resulting pressure is obtained as

$\mathrm{P}=-\frac{\rho_{f}}{\mu}\left[\frac{1+C e^{2 \mu h_{1}}}{1-C e^{2 \mu h_{1}}}+\frac{e^{-2 \mu h_{2}}+1}{e^{-2 \mu h_{2}}-1}\right] \frac{\partial^{2} W}{\partial t^{2}}=\mathrm{Z}_{f 3} \frac{\partial^{2} W}{\partial t^{2}}$

Where $h_{1}$ and $h_{2}$ are fluid level on top of the plate and fluid level below the plate surface, respectively. In the case of floating plate on the fluid free surface (Fig. 3) the resulting pressure is calculated using Eq. (35) at $\mathrm{h}_{2}$ level

$\mathrm{P}=-\frac{\rho_{f}}{\mu}\left[\frac{e^{-2 \mu h_{2}}+1}{e^{-2 \mu h_{2}}-1}\right] \frac{\partial^{2} W}{\partial t^{2}}=\mathrm{Z}_{f 4} \frac{\partial^{2} W}{\partial t^{2}}$

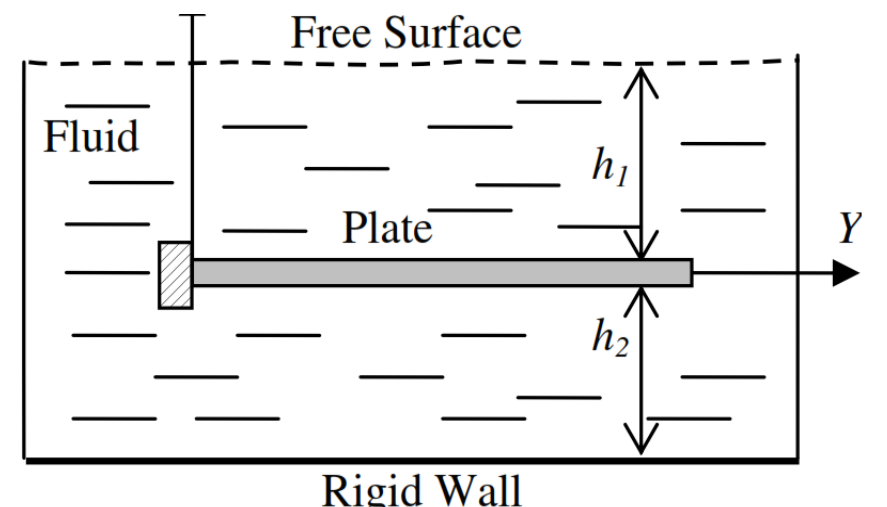

Figure 3: Boundary conditions of rectangular plate totally submerged in fluid [49].

\subsection{Boundary conditions}

The fluid domain is divided into two region as shown in fig.4. A constant velocity $u$ is imposed on the left side whereas right side set as outflow region at zero gradient value. Pressure on the both side set as atmospheric i.e. $\mathrm{P}=\mathrm{P}_{\mathrm{atm}}$

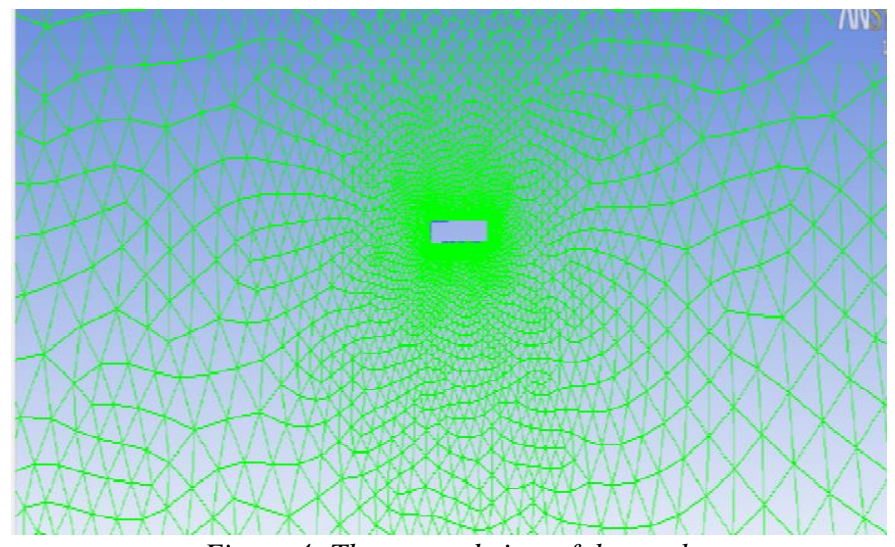

Figure 4: The zoomed view of the mesh

\subsection{Validation Case}

In order to validate the current numerical solver, simulations of flow past a flat plate were performed and compared to the published results of B.T. Tan et al., (1998). The validation results give a satisfactory measure of confidence in the fidelity of the simulation.

Table 1: Validation
\begin{tabular}{|c|c|c|c|}
\hline Validation & $\mathrm{C} / \mathrm{t}$ & $\begin{array}{c}\text { Reynolds } \\
\text { No }\end{array}$ & $\begin{array}{c}\text { Drag } \\
\text { Coefficient }\end{array}$ \\
\hline Present Result & 5 & 1000 & 0.561 \\
\hline B.T. Tan et al., (1998) [50] & 5 & 1000 & 0.555 \\
\hline
\end{tabular}

\section{Results and Discussion}

In steady flow, the pressure contours depict a different pressure throught the whole flat plate section at a particular Reynolds Number and a particular angle of attack.The pressure distribution changes with respect to different Reynolds number and different angle of attack.

In steady flow, the streamlines depict a different vortex formation throughout the whole flat plate section. The vortex is formed at the low pressure sites. In the streamline at $\mathrm{t}=5$ and $\mathrm{t}=10$ the vortex is formed at the same position and there is no change in the vortex number and the vortex size that is the number of vortex formed is four and the size of all the four vortices is same.

\subsection{Effect of angle of attack}

Numerical analyses are conducted to evaluate the `aerodynamic performance of uniform flow past a two dimensional flat plate at a chord Reynolds number of 500, 1000, 2000, and 3000 with angle of attack $0^{0}$ to $15^{\circ}$. The mean drag and lift force coefficients pertaining to their respective flat geometry are tabulated in table 2. At zero incidence, the drag production leads to some interesting observations. As expected, the overall drag coefficient of flat plate decreases as angle of attack increases because the viscous effects are more dominant at lower Reynolds numbers which cause the skin friction to be the major contributor to the overall drag. As it can be clearly seen that on increasing AOA from $0^{0}$ $15^{0}$ the area of the vortex formed in pressure contour as well as in streamline diagram is increasing, depicting that the coefficient of lift is increasing. Thus, for a steady flow, on increasing AOA the coefficient of lift increases but coefficient of drag is decreases.

\subsection{Effect of Reynolds Number in steady flow}

Now, if we talk about the effect of Reynolds number as we can see that on increasing the Reynolds number, the number of vortex forming is increasing, depicting increase in coefficient of lift. Thus, for a steady flow, coefficient of lift increases on increasing Reynolds number and coefficient of drag is decreases. Fig.5 shows the measured pressure gradient around the flat plate at Reynold No 500 and angle of attack $0^{0}$ to $15^{0}$ flat plate was designed to have a large leading-edge radius to flatten the peak in pressure coefficient near the plate tip to discourage flow separation, flow separation was still found to take place on the lifting surface even when the angle of attack varies from 0 to $15^{\circ}$ because of the low Reynolds numberA large circulation 
bubble was generated on the tip of the plate as a result of the flow separation. As angle of attack increasing, the pressure gradient over the surface of the plate become bigger and bigger. Therefore, the separation regions over the upper surfaces of flat plate enlarged significantly when the angle of attack increased to $15^{\circ}$.

Table 2: Values of $C D, C L$ gliding ratio and deflecting for different Reynolds Number with their AOA

\begin{tabular}{|c|c|c|c|c|c|}
\hline Reynolds No & AOA & $\begin{array}{c}\text { Lift coefficient } \\
\left(\mathrm{C}_{\mathrm{L}}\right)\end{array}$ & $\begin{array}{c}\text { Drag coefficient } \\
\left(C_{D}\right)\end{array}$ & $\mathrm{C}_{\mathrm{L}} / \mathrm{C}_{\mathrm{D}}$ & Deflection (mm) \\
\hline \multirow{4}{*}{500} & 0 & 0.026 & 1.446 & 0.01798 & 0.457 \\
\hline & 5 & 0.0376 & 1.39 & 0.02705 & 0.258 \\
\hline & 10 & 0.072 & 1.284 & 0.056075 & 0.223 \\
\hline & 15 & 0.1074 & 1.308 & 0.08211 & 0.229 \\
\hline \multirow{4}{*}{1000} & 0 & 0.002 & 0.5558 & 0.003598 & 0.146 \\
\hline & 5 & 0.0025 & 0.5314 & 0.004705 & 0.259 \\
\hline & 10 & 0.0014 & 0.6455 & 0.002169 & 0.261 \\
\hline & 15 & 0.071 & 0.666 & $2.29 \mathrm{E}-04$ & 0.242 \\
\hline \multirow{4}{*}{2000} & 0 & 0.001 & 0.3393 & 0.002947 & 0.231 \\
\hline & 5 & 0.018 & 0.3351 & 0.053715 & 0.201 \\
\hline & 10 & 0.0387 & 0.3514 & 0.110131 & 0.232 \\
\hline & 15 & 0.0559 & 0.3259 & 0.171525 & 0.234 \\
\hline \multirow{4}{*}{3000} & 0 & 0.0004 & 0.2406 & 0.001663 & 0.426 \\
\hline & 5 & 0.02 & 0.2291 & 0.087298 & 0.357 \\
\hline & 10 & 0.0389 & 0.2446 & 0.159035 & 0.386 \\
\hline & 15 & 0.0565 & 0.2241 & 0.25212 & 0.690 \\
\hline
\end{tabular}

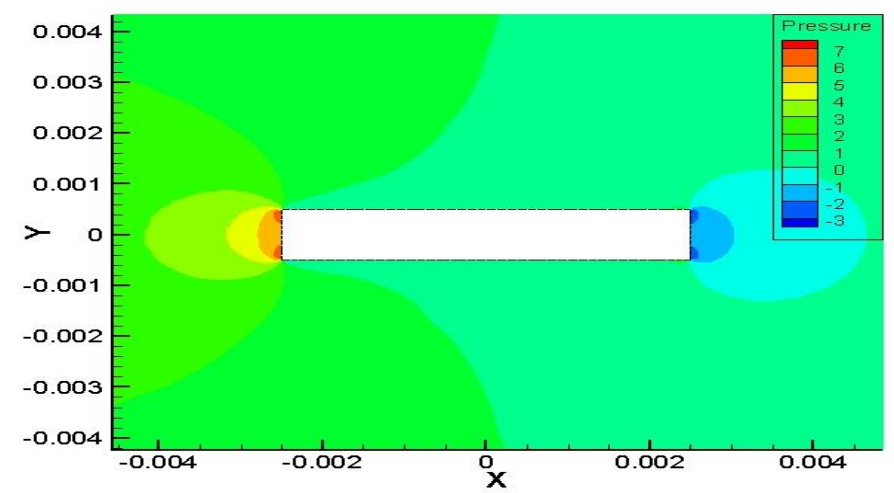

(a)

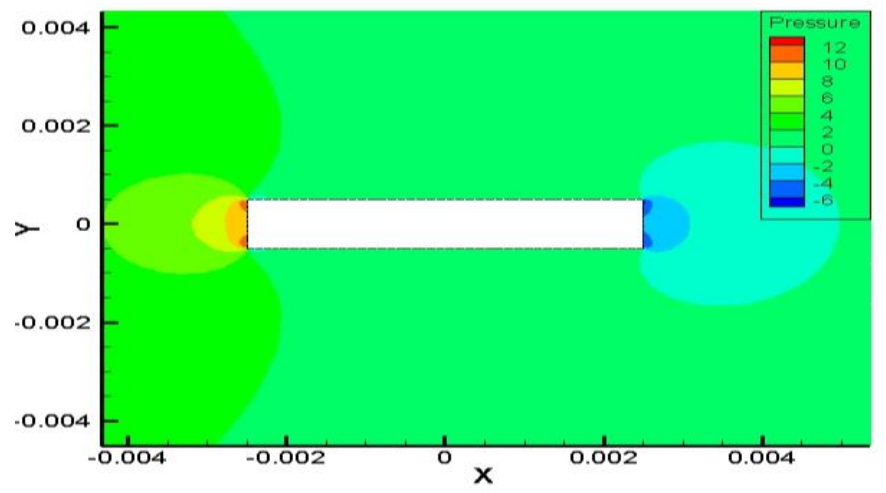

(c)

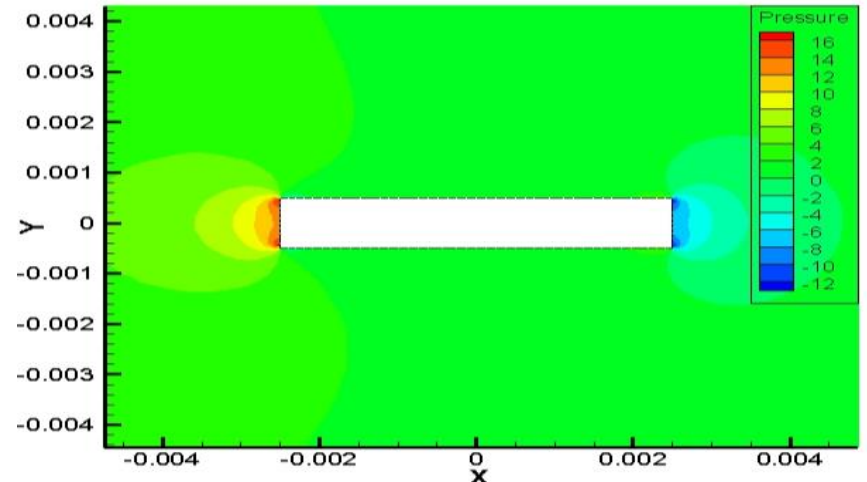

(b)

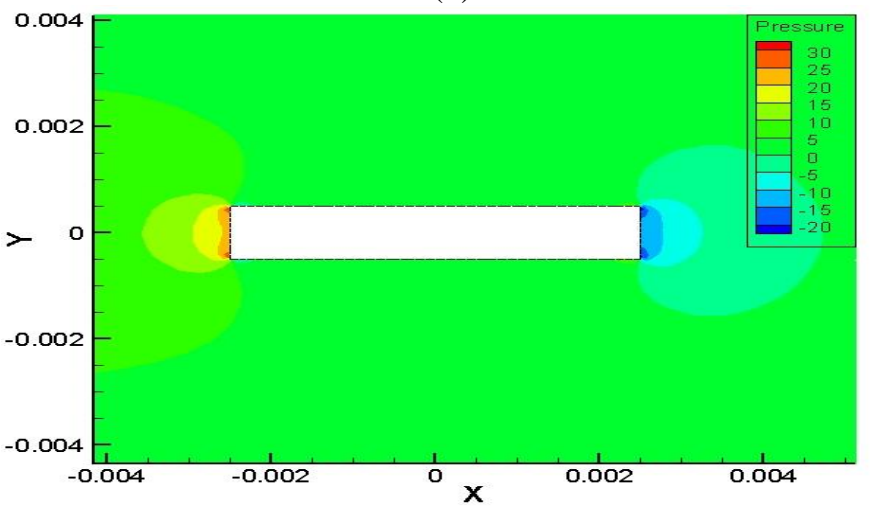

(d)

Figure 5: The pressure contour at Reynolds number 500 and angle of attack (a) $0^{\circ}$, (b) $5^{\circ}(\mathrm{c}) 10^{\circ}$ (d) $15^{\circ}$ 


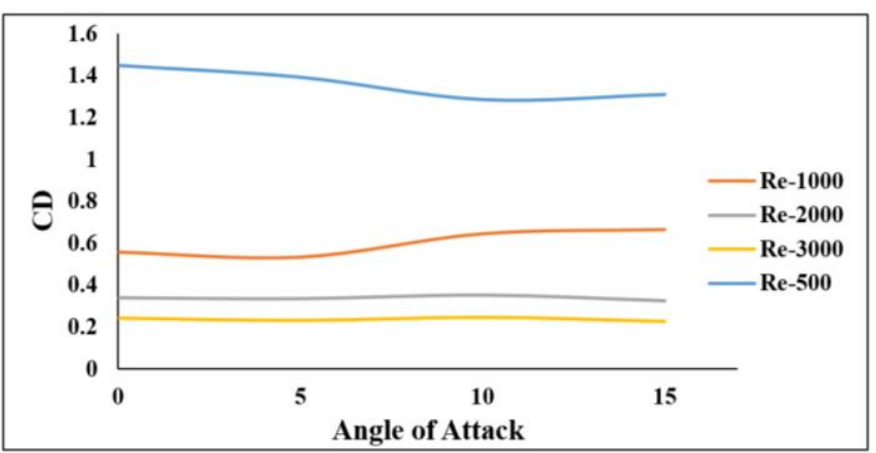

Figure 6: Variation of $C D$ with different angle of attack for different Reynolds number

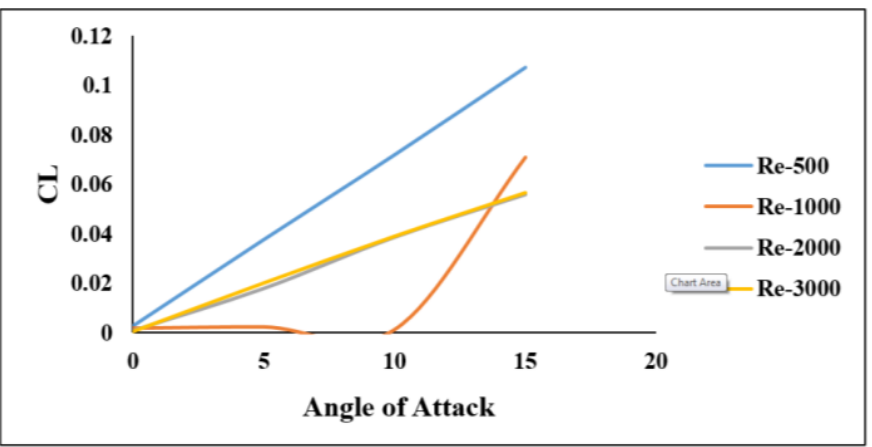

Figure 7: Variation of CL with different angle of attack for different Reynolds number

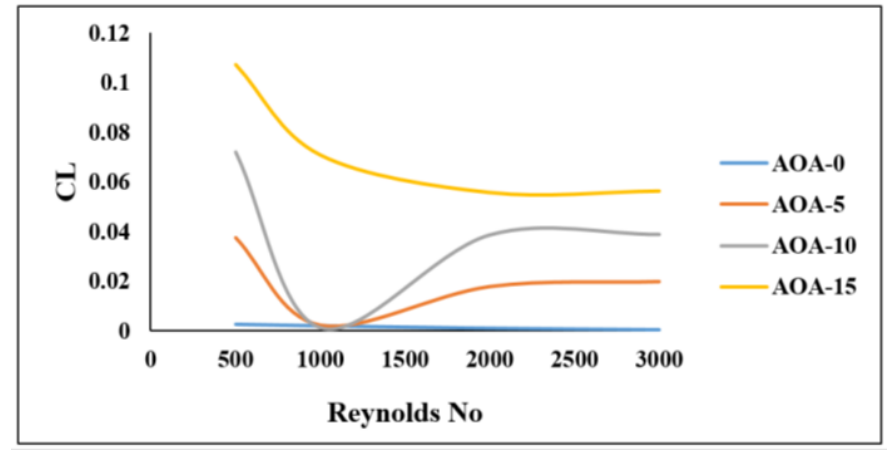

Figure 8: Variation of CL with different Reynolds number for different angle of attack

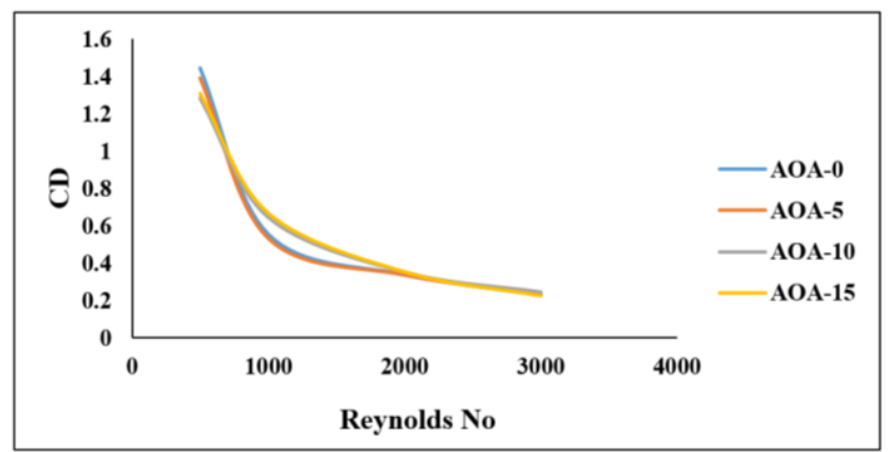

Figure 9: Variation of CD with different Reynolds number for different angle of attack

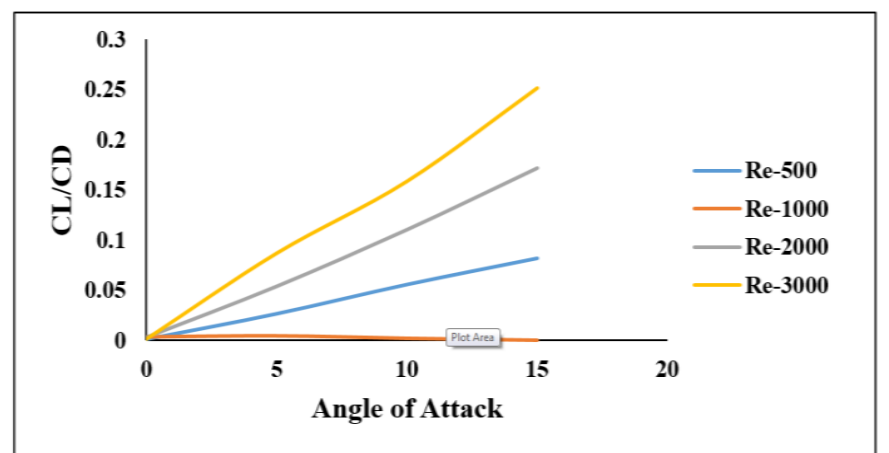

Figure 10: Variation of $\mathrm{Cl} / \mathrm{Cd}$ with different angle of attack for different Reynolds number

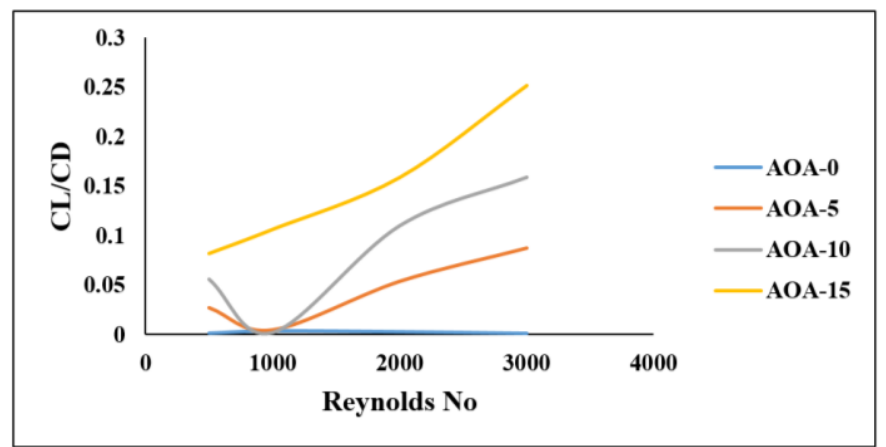

Figure 11: Variation of Cl/Cd with different Reynolds number for different angle of attack

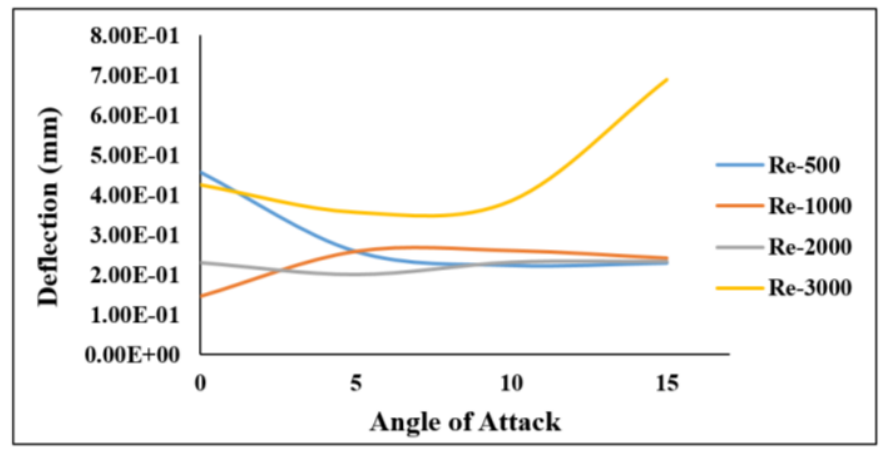

Figure 12: Variation of Deflection with different angle of attack for different Reynolds number

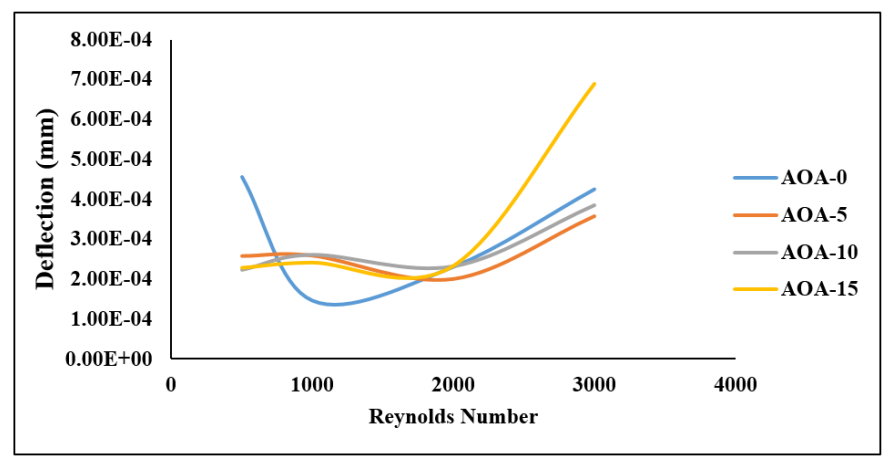

Figure 13: Variation of Deflection with different Reynolds number for different angle of attack 
A comparison of the time-mean force coefficient and gliding ratio with varying chord Reynolds numbers are shown in fig (6-11). At $\mathrm{Re}=500$, the lift for flat plate is seen to be increases from angle of attack $0^{0}$ to $15^{0}$, while drag of the flat plate decreases between these range. The flat plate experiences a greater rate of increases and generate the most CL Analyzing the coefficient of drag, one observes that the viscous effects are dominant at a Reynolds number below 3000 and the drag production for flat plate are decreasing. The effect of the Reynolds number on the gliding ratio is shown in table 2 . The maximum value of lift coefficient obtained at Re-1000 at angle of attack 150 whereas minimum lift was obtained at Re-1000 at AOA- $0^{0}$.

The result in the form of lift and drag forces are then fed into the ANSYS Workbench solver and coupling from fluid to structure has been performed. Fig. 12-13 shows the variation of deflection to Reynolds no and angle of attack. It is seen to be that deflection is decreases at Re-500 and angle of attack $\left(0^{0}\right.$ to $\left.15^{\circ}\right)$ but there is some interesting result was found between Re-1000 to 3000 at AOA- $0^{0}$ to $15^{\circ}$. The minimum deflection occur in Re-1000, angle of attack $0^{0}$ i.e. $0.146 \mathrm{~mm}$ and maximum value of deflection occurs in Re-3000 with angle of attack $15^{0}$ i.e. $0.690 \mathrm{~mm}$.

\section{Conclusions}

Numerical Analysis have been used to study the fluid structure interaction on a flat plate and simulations confirm the notion that at Steady flow is found for Re-500, 1000, 2000 and 3000 with AOA $0^{0}, 5^{0}, 10^{0}$ and $15^{0}$. The overall drag coefficient decreases as Re is increased. But it shows variations with the different angle of attack. Minimum coefficient of Drag is obtained at AOA $15^{0}$ for Reynolds number 3000 i.e. 0.224 and Minimum coefficient of lift is obtained at AOA $0^{0}$ for Reynolds number 3000 i.e. 0.0004 . Minimum Gliding ratio is obtained at AOA $-0^{0}$ for Reynolds number 3000 i.e. 0.001663 and Maximum Gliding ratio is obtained at $\mathrm{AOA}-15^{0}$ for Reynolds number 3000 i.e. 0.25212.Maximum Deflection is obtained at same AOA $-15^{0}$ for same Reynolds number 3000 i.e. $0.690 \mathrm{~mm}$.

\section{References}

[1] A.W. Leissa. Vibration of plates, NASA SP-160, Office of Technology Utilization, Washington, DC, 1969.

[2] J.N. Reddy, R.A. Arciniega, Shear deformation plate and shell theories: from Stavsky to present, Mech. Adv. Mater. Struct. 11 (2004) 535-582.

[3] E. Reissner, The effect of transverse shear deformation on the bending of elastic plates, J. Appl. Mech.-T ASME 12 (1945) 69-77.

[4] R.D. Mindlin, Influence of rotatory inertia and shear in flexural motion of isotropic elastic plates, J. Appl. Mech.-T ASME 18 (1951) 31-38.

[5] R.D. Mindlin, H. Deresiewicz, Thickness-shear and flexural vibrations of a circular disk, J. Appl. Phys. 25 (1954) 1329-1332.

[6] R.D. Mindlin, A. Schaknow, H. Deresiewicz, Flexural vibration of rectangular plates, J. Appl. Mech.-T ASME 23 (1956) 430-436.

[7] S.H. Hosseini Hashemi, M. Arsanjani, Exact characteristic equations for some of classical boundary conditions of vibrating moderately thick rectangular plates, Int. J. Solids Struct. 42 (2005) 819-853.

[8] H.C. Chen, R.L. Taylor, Vibration analysis of fluid solid systems using a finite element displacement formulation, International Journal of Numerical Methods in Engineering 29 (1990) 683-698.
[9] G. Fenves, L.M. Vargas-Loll, Nonlinear dynamic analysis of fluid-structure systems, Journal of Engineering Mechanics, American Societyof Civil Engineers 114 (1988) 219-240.

[10] L.G. Olson, K.J. Bathe, Analysis of fluid-structure interactions. A direct symmetric coupled formulation based on the fluid velocitypotential, Computers \& Structures 21 (1985) 21-32.

[11] O.C. Zienkiewicz, P. Bettes, Fluid-structure dynamic interaction and wave forces. An introduction to numerical treatment, International Journal of Numerical Methods in Engineering 13 (1978) 1-16.

[12] O.C. Zienkiewicz, R.E. Newton, Coupled vibration of a structure submerged in a compressible fluid, International Symposium on Finite Element Techniques, Stuttgart, Germany, 1969.

[13] W.C. M.uller, Simplified analysis of linear fluid-structure interaction, International Journal of Numerical Methods in Engineering 17 (1981) 113121.

[14] G. Sandberg, P. G . oransson, Asymmetric finite element formulation for acoustic fluid-structure interaction analysis, Journal of Sound and Vibration 123 (3) (1988) 507-515.

[15] G. Sandberg, A new strategyfor solving fluid-structure problems, International Journal of Numerical Methods in Engineering 38 (1995) $357-$ 370.

[16] O.C. Zienkiewicz, D.K. Paul, E. Hinton, Cavitation in fluid-structure response (with particular reference to dams under earthquake loading), Earthquake Engineering \& Structural Dynamics 11 (1983) 463-481.

[17] A. Bermudez, R. Dur ! an, M.A. Muschietti, R. Rodr ! 1guez, J. Solomin, Finite element vibration analysis of fluid- solid systems without spurious modes, SIAM Journal of Numerical Analysis 32 (1995) 1280-1295.

[18] D. Maity, S.K. Bhattacharyya, Response of structural system in fluid environment, in: P.M. Mujumdar, A. Joshi (Eds.), Advances in Testing, Design, and Development of Aerospace Structures, Proceedings of the Fifth National Seminar on Aerospace Structures, Indian Institute of Technology, Bombay, Allied Publishers Ltd., India, 1996, pp. 431-441.

[19] L.G. Olson, K.J. Bathe, A studyof displacement-based fluid finite elements for calculating frequencies of fluid and fluid-structure systems, Nuclear Engineering and Design 76 (1983) 137-151.

[20] A.K. Chopra, P. Chakraborti, Earthquake analysis of concrete gravity dams including dam-water-foundation rock interaction, Earthquake Engineering \& Structural Dynamics 9 (1981) 363-383.

[21] J.F. Hall, A.K. Chopra, Two-dimensional dynamic analysis of concrete gravity and embankment dams including hydrodynamic effects, Earthquake Engineering \& Structural Dynamics 10 (1982) 305-332.

[22] M.K. Au-Yang, J.E. Galford, Fluid-structure interaction-a survey with emphasis on its application to nuclear steam system design, Nuclear Engineering and Design 70 (1982) 387-399.

[23] I. Antoniadis, A. Kanarachos, Decoupling procedures for fluid-structure interaction problems, Computer Methods in Applied Mechanics and Engineering 70 (1988) 1-25.

[24] R.K. Singh, T. Kant, A. Kakodkar, Coupled shell-fluid interaction problems with degenerate shell and three dimensional fluid elements, Computers \& Structures 38 (1991) 515-528.

[25] H. Lamb, On the vibrations of an elastic plate in contact with water, Proc. Roy. Soc. A 98 (1921) 205-216.

[26] J.H. Powell, J.H.T. Roberts, On the frequency of vibration of circular diaphragms, Proc. Phys. Soc. Lond. 35 (1923) 170-182.

[27] U.S. Lindholm, D.D. Kana, W.H. Chu, et al., Elastic vibration characteristics of cantilever plates in water, J. Ship Res. 9 (1) (1965) $11-22$.

[28] G.C. Volcy, P. Morel, M. Bureau, et al., Some studies and researches related to the hydro-elasticity of steel work, in: Proceedings of the 122nd Euromech Colloquium on numerical analysis of the dynamics of ship structures, Ecole Polytechnique, Paris, 1979, pp. 403-406.

[29] Y. Fu, W.G. Price, Interactions between a partially or totally immersed vibrating cantilever plate and the surrounding fluid, J. Sound Vibrat. 118 (3) (1987) 495-513.

[30] Husain Mehdi, Fahad Anwer, Akhlaque Ahmad,"Fluid Structure Interaction of Flow around a Pleated Insect 2D Airfoil at Ultra Low Reynolds Numbers" International Journal of Research in Aeronautical and Mechanical Engineering, vol.3, issue 3, pp 19-37, 2015. 
[31] Syed Fahad Anwer, Intesaaf Ashraf, Husain Mehdi, Akhlaq Ahmad " On the Aerodynamic Performance of Dragon fly wing Section in Gliding Mode" Advances in Aerospace Science and Applications, Vol. 3, pp 227-234, 2013.

[32] Husain Mehdi, Brajesh Kumar, Anil Kamboj"Numerical Analysis of Steady and Unsteady Flow for Dragonfly Wing Section in Gliding Mode" International Journal of Advanced Mechanical Engineering, Vol.4, pp 365370,2014

[33] Husain Mehdi, Fahad Anwer, Akhlaque Ahmad, "Vibration Analysis of Dragonfly wing section in Gliding Mode at Low Reynolds Number" International Journal of Research in Aeronautical and Mechanical Engineering, vol. 2, issue 12, pp 11-23, 2014.

[34] Husain Mehdi, Vipul Namdev, Prashant Kumar, Ashish Tyagi, "Numerical Analysis of Fluid Flow around a Circular Cylinder at Low Reynolds Number", IOSR, Journal of Mechanical and Civil Engineering, vol 3, issue 3, 2016, 94-101.

[35] Husain Mehdi, Shwetanshu Gaurav, Mudit Sharma, Numerical Investigation of Fluid Flow and Aerodynamic performance on a 2D NACA4412 Airfoil, International Journal of Research in Engineering and Innovation Vol-1, Issue-1 (May-2017), 1-5.

[36] J.L. Sanders, An improved first approximation theory for thin shell, NASA TR-24 (1959).

[37] E. Reissner, A new derivation of the equation for the deformation of elastic shells, Am. J. Math. 63 (1941) 177-184.

[38] V.S. Vlasov, Basic differential equations in general theory of elastic shells, NACA-TM-1241 (1951) 59

[39] S. Timoshenko, Theory of Plates and Shells, McGraw-Hill, New York, 1959.
[40] Y. Kerboua, A.A. Lakis, M. Thomas, L. Marcouiller, Vibration analysis of rectangular plates coupled with fluid, Applied Mathematical Modelling 32 (2008) 2570-2586.

[41] C.C. Liang, C.C. Liao, et al., The free vibration analysis of submerged cantilever plates, Ocean Eng. 28 (2001) 1225-1245.

[42] M.H. Toorani, A.A. Lakis, Dynamic analysis of anisotropic cylindrical shells containing flowing fluid, Transactions of the ASME, J. Pressure Vessel Technol. 123 (4) (2001) 454-460.

[43] Y.K. Cheung, D. Zhou, Hydroelastic vibration of a circular container bottom plate using the Galerkin method, J. Fluid Struct. 16 (4) (2002) 561-580.

[44] M.K. Kwak, S.B. Han, Effect of fluid depth on the hydroelastic vibration of free-edge circular plate, J. Sound Vibrat. 230 (1) (2000) 171-185.

[45] C.C. Liang, C.C. Liao, et al., The free vibration analysis of submerged cantilever plates, Ocean Eng. 28 (2001) 1225-1245.

[46] Y.K. Cheung, D. Zhou, Coupled vibratory characteristics of a rectangular container bottom plate, J. Fluid Struct. 14 (2000) 339-357.

[47] A. Ergin, B. Ugurlu, Linear vibration analysis of cantilever plates partially submerged in fluid, J. Fluid Struct. 17 (2003) 927-939.

[48] M.H. Meylan, The forced vibration of a thin plate floating on an infinite liquid, J. Sound Vibrat. 205 (5) (1997) 581-591.

[49] M.H. Toorani, A.A. Lakis, General equations of anisotropic plates and shells including transverse shear deformations, rotary inertia and initial curvature effects, J. Sound Vibrat. 237 (3) (2000) 561-615.

[50] B.T. Tan, M.C. Thompson, K. Hourigan, Simulated Flow around Long Rectangular Plates under Cross Flow Perturbations, International Journal of Fluid Dynamics, Vol. 2 (1), 1998.

Cite this article as: Abhishek Yadav, Arshad Mehmood, Numerical analysis and fluid structure interaction of flow around a flat plate at low reynolds number, International Journal of Research in Engineering and Innovation Vol-4, Issue-1 (2020), 60-68.

https://doi.org/10.36037/IJREI.2020.4106. 